\title{
A MECHANOCHEMICAL MODEL OF ANGIOGENESIS AND VASCULOGENESIS
}

\author{
DAPHNE MANOUSSAKI ${ }^{1}$
}

\begin{abstract}
Vasculogenesis and angiogenesis are two different mechanisms for blood vessel formation. Angiogenesis occurs when new vessels sprout from pre-existing vasculature in response to external chemical stimuli. Vasculogenesis occurs via the reorganization of randomly distributed cells into a blood vessel network. Experimental models of vasculogenesis have suggested that the cells exert traction forces onto the extracellular matrix and that these forces may play an important role in the network forming process. In order to study the role of the mechanical and chemical forces in both of these stages of blood vessel formation, we present a mathematical model which assumes that (i) cells exert traction forces onto the extracellular matrix, (ii) the matrix behaves as a linear viscoelastic material, (iii) the cells move along gradients of exogenously supplied chemical stimuli (chemotaxis) and (iv) these stimuli diffuse or are uptaken by the cells. We study the equations numerically, present an appropriate finite difference scheme and simulate the formation of vascular networks in a plane. Our results compare very well with experimental observations and suggest that spontaneous formation of networks can be explained via a purely mechanical interaction between cells and the extracellular matrix. We find that chemotaxis alone is not a sufficient force to stimulate formation of pattern. Moreover, during vessel sprouting, we find that mechanical forces can help in the formation of well defined vascular structures.
\end{abstract}

Mathematics Subject Classification. 74H15, 92C10, 92C15, 92C17.

\section{INTRODUCTION}

The first blood vessels form during development as randomly distributed cells reorganize to form aggregates and subsequently a network of vessels. The process of this initial vascular network formation is called vasculogenesis $[7,8]$. As the organism develops, subsequent growth of the vascular network occurs mainly via angiogenesis, whereby new vessels sprout from existing vessels into the surrounding tissue $[22,23]$.

Angiogenesis has been shown to be chemotactically-driven. In response to an angiogenic stimulus, the endothelial cells that line blood vessels, will sprout from the existing vessels grow towards the source of the stimulus forming a new vessel. This mechanism occurs also during tumor vascularization: new vessels sprout from the neighbouring vasculature in response to chemical factors secreted by the tumor.

While chemotaxis, i.e. motion of cells up chemical concentration gradients, is important during angiogenesis, mechanical cell-ECM interactions may play a significant role during vasculogenesis.

Keywords and phrases. Angiogenesis, vasculogenesis, chemotaxis, extracellular matrix, theoretical models, numerical solution.

1 Department of Applied Mathematics, University of Crete, 71409 Heraklion, Greece. e-mail: daphne@tem.uoc.gr 
In vitro models of vasculogenesis have shown that growth factors (VEGF-A) are required during these initial stages of blood vessel formation but contractile forces that endothelial cells exert onto their surrounding extracellular collagen material (ECM - extracellular matrix) may also play an important role in the formation of such vascular networks [25,27]. Mechanical interactions between cells and the ECM occur also in other processes of vascular morphogenesis: During the development of the epicardiac cushion and heart, for example, Markwald et al. [17], suggested that these structures appear in the embryo as the cardiac gelly is organized by endothelial cells in "tracks" that subsequently serve as migratory pathways for the cells.

In order to study the factors influencing blood vessel formation, a number of mathematical models have been presented $([10,14,24]$ or, for a review of earlier work see, for example, [4]). In these models blood vessel formation has been studied in relation to cell motion along gradients of chemical concentration and/or adhesiveness. The possible effects of cell forces during tumor-induced angiogenesis were explored in [12].

In $[15,16]$ we presented a mathematical model for vasculogenesis, which was the first model to show that mechanical forces between cells and their surrounding collagen material could play the main role in the reorganization of cells into a vascular network. We showed that the ECM is reorganized and the vascular networks form as a result of the traction forces exerted by the cells onto the ECM, and that neither cell migration nor anisotropic cell behavior are necessary for the pattern to form.

In this paper, we present a mathematical model that describes mechanical and chemical interactions during blood vessel formation and study some of the effects of mechanochemical forces during angiogenesis and vasculogenesis. The model is based on the theory presented by Murray, Oster and Harris [20,21] and it is an extension of the mechanical model describing vasculogenesis presented by Manoussaki et al. in [15,16,19], as it also considers the chemical interaction between cells and sources of angiogenic stimuli. It consists of a system of non-linear partial differential equations which include advection, diffusion and reaction terms. In particular:

- the cells are described as a population density that moves with velocities that are triggered either by chemical gradients, random motion or by advection. If cell growing is also taken into consideration, cells are modeled by a reaction-advection-diffusion equation;

- the collagen material is assumed to be a linear viscoelastic material which deforms under cell-exerted stress;

- chemical production and uptake by the cells s modeled via a reaction-diffusion equation.

Reaction-diffusion and chemotaxis processes are found in many biological systems and have been studied analytically and numerically. However, as the mechanical aspects of the interactions between cells and their ECM have been emphasized only in recent years, the techniques for studying the corresponding mathematical models are less developed.

The equations of the present model contain nonlinear terms and are too difficult to solve analytically. In order to study the model and test its pattern-forming capabilities, we resort to a numerical study of the equations. We present a scheme for discretizing the equations using finite differences. In the discretization the different dynamics of the equations (reaction-advection-diffusion) are calculated using a fractional step method, whereby equations are solved by integrating each of the reaction, advection, diffusion terms separately.

Advection terms are solved using slope-limiter methods and stiff equations using Crank-Nicholson. Each method separately is known and well studied, but they have not been applied to a similar set of equations before and the methodology presented here appears to yield results which compare very well with experimentally observed cellular networks.

\subsection{Biological background}

Endothelial cells are the key player in formation of blood vessels. Angioblasts, precursor cells to endothelial cells, will reorganize within the embryo to eventually make the first vascular network via vasculogenesis. Subsequently, new vessels will form as endothelial cells sprout from existing vasculature, in resonse to external stimuli. The cells will then migrate into the neighbouring tissue and join with other cells to form new capillaries.

In vitro angiogenesis systems have been developed in order to study the vessel forming potential of cells in a controled manner [9]. In such systems endothelial cells (as well as other cell types, such as fibroblasts) are 
placed on a layer of a collagen gel (extracellular matrix-ECM). Matrigel, a commercially available such gel, is often used in angiogenesis assays.

In in vitro models of vasculogenesis, cells are initially seeded randomly onto the ECM and they subsequently reorganize into networks. During such experiments it was shown that when endothelial cells are seeded onto a plane of Matrigel, they attach onto it and exert traction forces onto the ECM [25,27]. Pulling causes not only the ECM to move, but also the cells that have adhered onto the moving gel. After a few hours, the cells appear to form aggregates and the ECM appears to have become reorganized: most of it is accumulated underneath cell clusters, while the remaining ECM has reorganized into a network of fibrous lines that tesselate the plane. These matrical lines run between cell clusters and are used by the cells as a scaffold for their migration. The cells progressively move from the clusters onto the adjoining lines, fill the lines, and eventually form a network of cellular cords [25].

During development, subsequent remodelling of the vascular plexus depends on cellular proliferation and reaction of the cells to chemical and mechanical stimuli. In particular, in response to concentration gradients of angiogenic chemicals, (such as vascular endothelial growth factors), endothelial cells will degrade the adjacent mesh-like basal lamina that surrounds a blood vessel, will sprout and grow towards the source of the angiogenic stimulus, and new vessels will join together at the tips (anastomose) to form vascular loops. In the case of tumors, vessels are elicited from the peripheral vasculature of the healthy tissue in response to gradients of tumor secreted TAF (tumor angiogenesis factor).

While mechanical interactions appear to be key in the development of the initial vasculature, their role in subsequent blood vessel sprouting and growth is less clear. Mathematical modeling can describe different possible interactions in a cell-ECM environment and can help in testing the pattern forming capabilities under different hypotheses.

\section{Mathematical MOdel}

The formation of pattern will be quantified in terms of changes in the local cell density, amount of ECM and ECM deformation. We use $n(\mathbf{x}, t)$ to denote the density of cells at time $t$ and on a point $\mathbf{x}$ in space. Cells apply forces to the ECM, so that each point of the ECM gets displaced by $\mathbf{u}=\left(u_{1}, u_{2}\right)$ from its original location $\mathbf{x}_{0}$, where $\mathbf{u}=\mathbf{x}(t)-\mathbf{x}_{0}$. These displacements create a strain field $\epsilon$ (approximated for small strains by $\left.\epsilon=\frac{1}{2}\left(\nabla \mathbf{u}+\nabla \mathbf{u}^{T}\right)\right)$ which can affect cellular movement. Cells, moreover, will respond to gradients of angiogenic factors and will move up such gradients. The chemical concentration we will denote by $c(\mathbf{x}, t)$, while the amount of ECM by $\rho(\mathbf{x}, t)$.

Note should be given in the distinction between biological cells and computational cells, which will be mentioned later, as well as between the collagen substratum (extracellular-collagen-matrix), that will also be refered to as "matrix", which is, of course, different to the mathematical matrix.

\subsection{Endothelial cell conservation equation}

Local changes in cell density will take place as cells proliferate, die or move to neighbouring locations:

$$
\frac{\partial n}{\partial t}+\nabla \cdot J_{\text {cell }}=r n
$$

where the right hand term assumes a density-dependent growth of the cell population and $r$ is the intrinsic growth rate of the cells.

$J_{\text {cell }}$ denotes the cellular flux. Depending on the biological assumtions, cell migration can depend on chemical gradients (chemotaxis), on the strain of the collagen substratum, or the cells will move passively, with velocity 
$\mathbf{v}=\frac{d}{d t} \mathbf{u}$, if they adhere onto a deforming substratum (advection):

$$
\begin{aligned}
& J_{\text {cell }}=-\nabla \cdot(D(\epsilon) n)+n \chi(c) \nabla c+n \frac{d \mathbf{u}}{d t}, \\
& \text { Flux }=\text { strain-dependent movement }+ \text { chemotaxis }+ \text { passive advection } .
\end{aligned}
$$

The term $\chi(c)=\frac{\chi_{0}}{1+\beta c}$ is a form that is presented in [5] and it assumes that chemical gradients take effect when the chemical concentration is low; for high concentrations the corresponding receptors saturate and cannot feel the gradient. $D(\epsilon)$ is a tensor describing strain-dependent movement and the form used here is presented below.

\subsubsection{Strain-dependent movement}

In the absense of chemotactic movement, endothelial cells that were cultured on Matrigel, were observed to leave clusters, where we assume collagen fibres have an orientation that is random, and move towards and onto the matrix lines, that consisted of aligned collagen fibers [25]. We model this movement as a cellular movement is a random, but biased towards the direction of principal strain.

The diffusion coefficient $D(\epsilon)$ is thus a tensor that depends on the strain $\epsilon$ and describes the preferential movement of the cells along areas where fibers are compressed and aligned $[6,13]$ and is proportional in each direction to the degree of fiber alignment due to strain.

Cook [6] derives a form for $D$ using techiques presented by Advani and Tucker [1], and of his derivation we present a short summary (for details, see [6]). $D$ is calculated by considering:

$$
D_{i j}=D_{1} \int f(\psi) \hat{\psi}_{i} \hat{\psi}_{j} d \psi
$$

where $D_{1}$ is a scalar parameter, $\hat{\psi}$ is the unit orientation vector $\hat{\psi}=\left[\begin{array}{ll}\cos \psi & \sin \psi\end{array}\right]^{T}$ and $f(\psi)$ is the orientation distribution of the fibres under a transformation $M$.

When $M=I-\nabla_{\mathbf{y}} \mathbf{u}$ represents the transformation matrix of a small element of the cell culture, from the deformed state (with coordinates $\mathbf{y}=\left(x_{2}, y_{2}\right)$ ), to the reference state (whose coordinates are $(x, y)$ ) and when the fibre orientation distribution is uniform in the reference state, then in the deformed state the orientation distribution of the fibres is given by

$$
f(\psi)=\frac{1}{2 \pi} r^{2}|M|
$$

The stretch factor $r=r(\psi)$ for the deformation corresponding to an angle $\psi$ is given by

$$
r^{2}=\frac{1}{\hat{\psi}^{T} M^{T} M \hat{\psi}}
$$

It is derived by considering that the radius of a unit circle is rotated from an angle $\theta$ to an angle $\psi$ and stretched from length 1 to length $r$ via the transformation $M^{-1}$, so that

$$
M^{-1} \hat{\theta}=r \hat{\psi}
$$

We thus have that $1=\hat{\theta}^{T} \hat{\theta}=r^{2} \hat{\psi}^{T} M^{T} M \hat{\psi}$ from which equation (2) follows.

For small strains equation (1) takes the form

$$
D(\epsilon)=\hat{D}_{1}\left(\begin{array}{cc}
2+\epsilon_{11}-\epsilon_{22} & \epsilon_{12}+\epsilon_{21} \\
\epsilon_{12}+\epsilon_{21} & 2-\epsilon_{11}+\epsilon_{22}
\end{array}\right),
$$

where $\epsilon_{11}, \epsilon_{12}, \epsilon_{21}, \epsilon_{22}$ are the strains in the individual directions and $\hat{D}_{1}=\frac{D_{1}}{2}$. 


\subsection{Matrix conservation equation}

In development the collagen matrix is produced by cells, and it is subsequently reorganized by them, as cells pull on it or migrate on it. In vitro there is often no significant production or degradation of matrix, and so we assume that local changes in matrix thickness occur just because the cells rearrange it on the culture dish:

$$
\frac{\partial \rho}{\partial t}+\nabla \cdot\left(\frac{d \mathbf{u}}{d t} \rho\right)=0
$$

In considering the above form we shall assume that in general matrix production or degradation plays a less important role in the patterning process.

\subsection{Force balance equation}

The substratum (collagen matrix) reorganizes as a result of the traction forces that the cells exert onto it. We consider the forces exerted by the cells are approximately in balance with the viscoelastic resistance offered by the matrix (pseudo- steady state) and external body forces:

$$
\nabla \cdot\left(\sigma_{\text {cell }}+\sigma_{E C M}\right)+F_{d r a g}=0
$$

The total traction exerted by the cells is proportional to the cell density

$$
\sigma_{\text {cell }}=\tau_{0} \frac{1}{1+\alpha n^{2}} \operatorname{In}
$$

where $\tau=\tau_{0} \frac{1}{1+\alpha n^{2}} I$ is the intrinsic traction per cell, which we assume is isotropic and that it saturates at high cell densities. We assume that the matrix responds to the traction forces as a linear viscoelastic material (Voigt body), so that the stress developed in the matrix when a strain $\epsilon$ is applied, is

$$
\sigma_{E C M}=\mu_{1} \epsilon_{t}+\mu_{2} \theta_{t} I+\frac{E}{\nu+1}\left(\epsilon+\frac{\nu}{1-2 \nu} \theta I\right) .
$$

Additionally, the resistance to the movement of the matrix across the domain is modeled as a viscous drag

$$
F_{d r a g}=-s \frac{1}{\rho} \frac{d u}{d t}
$$

giving the following equation for the balance of forces:

$$
\nabla \cdot\left(\tau_{0} \frac{1}{1+\alpha n^{2}} I+\mu_{1} \epsilon_{t}+\mu_{2} \theta_{t} I+\frac{E}{\nu+1}\left(\epsilon+\frac{\nu}{1-2 \nu} \theta I\right)\right)-s \frac{1}{\rho} \frac{d u}{d t}=0 .
$$

The following are constant model parameters: $\tau_{0}$ (traction per cell), $\alpha$ (a measure for cell traction saturation), $\mu_{1}, \mu_{2}$ (shear and bulk viscosities), $E$ (Youngs Modulus), $\nu$ (Poisson ratio), and $s$ measure of viscous drag.

\subsection{Endothelial activators}

Cells will also move along gradients of certain chemical factors (chemotaxis). For example, in tumor angiogenesis, formation of capillary sprouts are considered to be up gradients of tumor angiogenesis factor (TAF), which is also uptaken by the cells [3,11], or vascular endothelial growth factor (VEGF). Here, we assume that such an angiogenic factor $c(\mathbf{x}, t)$ is uptaken by the endothelial cells and diffuses. In the model allow also forits 
TABLE 1. Values of characteristic quantities.

\begin{tabular}{|l|l|l|}
\hline Parameter & Description & Value range \\
\hline$N$ & Average seeding cell density & $1.2-1.8 \times 10^{4}$ cells $/ \mathrm{cm}^{2}$ \\
\hline$\rho_{0}$ & Average matrix thickness & $10 \mu \mathrm{m}-1 \mathrm{~mm}$ \\
\hline$L$ & Characteristic length & $10^{-1}-10^{1} \mathrm{~mm}$ \\
\hline$T$ & Characteristic time scale & $1-20 \mathrm{~h}$ \\
\hline
\end{tabular}

potential secretion by the endothelial cells:

$$
\begin{aligned}
\frac{\partial c}{\partial t}= & D_{2} \nabla^{2} c+\gamma n \\
& \text { diffusion }+ \text { production by cells }- \text { uptake by cells, }
\end{aligned}
$$

where $\gamma$ is the rate of production of $c$ by the cells, while the uptake is governed by Michaelis-Menten kinetics [5].

\subsection{Dimensional analysis}

The model we analyze is:

$$
\begin{aligned}
n_{t}+\nabla \cdot\left(n \frac{d \mathbf{u}}{d t}\right) & =\nabla \cdot \nabla\left(D_{1}(\epsilon) n\right)-\nabla \cdot(n \chi(c) \nabla c)+r n, \\
\rho_{t}+\nabla\left(\rho \frac{d \mathbf{u}}{d t}\right) & =0, \\
s \frac{\mathbf{u}_{t}}{\rho} & =\nabla \cdot\left[\left(\mu_{1} \epsilon_{t}+\mu_{2} \theta_{t} I\right)+\frac{E}{1+\nu}\left(\epsilon+\frac{\nu \theta I}{1-2 \nu}\right)+\frac{\tau_{0} n I}{1+n^{2} \alpha}\right], \\
\frac{\partial c}{\partial t} & =D_{2} \nabla^{2} c+\gamma n-\delta \frac{n c}{K_{m}+c},
\end{aligned}
$$

where $\frac{d \mathbf{u}}{d t}=\mathbf{v}=\frac{\partial \mathbf{u}}{\partial t}+(\mathbf{v} \cdot \nabla) \mathbf{u}$ is the velocity of the matrix.

We introduce as non-dimensional variables:

$$
\begin{array}{rll}
n^{*}=n / N, & \mathbf{u}^{*}=\mathbf{u} / L, & \rho^{*}=\rho / \rho_{0}, \\
c^{*}=c / C, & t^{*}=t / T=t \frac{V}{L}, & x^{*}=x / L, \\
y^{*}=y / L, & \mathbf{v}^{*}=\mathbf{v} / V, & \epsilon^{*}=\epsilon
\end{array}
$$

We set the non-dimensional parameters:

$$
\begin{array}{ccc}
\hat{\mu}_{i}=\frac{\mu_{i}(1+\nu)}{T E}, & \hat{\tau}=\frac{\tau_{0} N(1+\nu)}{E}, & \hat{s}=\frac{s(1+\nu) L^{2}}{T E \rho_{0}}, \\
\hat{\nu}=\frac{\nu}{1-2 \nu}, & \hat{\alpha}=\alpha N^{2}, & d_{1}=\frac{L^{2}}{T}, \\
\hat{\chi}_{0}=\frac{T C}{L^{2}} \chi_{0}, & \hat{\beta}=\beta C, & \hat{r}=r T, \\
\hat{D}_{2}=D_{2} \frac{T}{L^{2}}, & \hat{\gamma}=\gamma \frac{N T}{C}, & \hat{\delta}=\delta \frac{N T}{C}, \\
\hat{K}_{m}=\frac{K_{m}}{C}, & \bar{D}^{*}=\hat{D}_{1} / d_{1} . &
\end{array}
$$


The non-dimensional form of the equations ((3)-(5)), dropping the asterisks on the non-dimensional variables for simplicity, are:

$$
\begin{aligned}
n_{t}+\nabla \cdot\left(\mathbf{u}_{t} n\right) & =\nabla \cdot \nabla \cdot(\bar{D}(\epsilon) n)-\nabla \cdot\left(\frac{\hat{\chi}_{0}}{1+\hat{\beta} c} n \nabla c\right)+\hat{r} n, \\
\rho_{t}+\nabla \cdot\left(\mathbf{u}_{t} \rho\right) & =0, \\
\hat{s} \frac{\mathbf{u}_{t}}{\rho} & =\nabla \cdot\left[\left(\hat{\mu}_{1} \epsilon_{t}+\hat{\mu}_{2} \theta_{t} I\right)+(\epsilon+\hat{\nu} \theta I)+\frac{\hat{\tau} n I}{1+\hat{\alpha} n^{2}}\right], \\
\frac{\partial c}{\partial t} & =\hat{D}_{2} \nabla^{2} c+\hat{\gamma} n-\hat{\delta} \frac{n c}{\hat{K}_{m}+c},
\end{aligned}
$$

with

$$
\bar{D}(\epsilon)=D\left[\left(1-\frac{\theta}{2}\right) I+\epsilon\right]=D\left(\begin{array}{cc}
1+\frac{\epsilon_{11}-\epsilon_{22}}{2} & \frac{\epsilon_{12}+\epsilon_{21}}{2} \\
\frac{\epsilon_{12}+\epsilon_{21}}{2} & 1-\frac{\epsilon_{11}-\epsilon_{22}}{2}
\end{array}\right) .
$$

Since $\mathbf{v}=\frac{d \mathbf{u}}{d t}=\mathbf{u}_{t}+\mathbf{v} \cdot \nabla \mathbf{u} \Rightarrow \mathbf{v} \cdot(I-\nabla \mathbf{u})=\mathbf{u}_{t}$, we can make the approximation $\frac{d \mathbf{u}}{d t} \approx \mathbf{u}_{t}$ for infinitesimal strains, an assumption which we used in equations $((7)-(9))$.

\subsection{The model}

Seeking numerical solutions, we drop the hat notation on the nondimensional parameters, and rewrite the model equations as:

$$
\begin{aligned}
& n_{t}+\left[u_{1 t} n+\chi(c) c_{x} n\right]_{x}+\left[u_{2 t} n+\chi(c) c_{y} n\right]_{y}=\left(D_{11} n\right)_{x x}+2\left(D_{12} n\right)_{x y}+\left(D_{22} n\right)_{y y}+r n, \\
& \rho_{t}+\left(u_{1 t} \rho\right)_{x}+\left(u_{2 t} \rho\right)_{y}=0 \\
& \frac{s}{\rho} u_{1 t}-\mu u_{1 x x t}-\frac{\mu_{1}}{2} u_{1 y y t}-(1+\nu) u_{1 x x}-\frac{1}{2} u_{1 y y}=\tau\left(\frac{n}{1+\alpha n^{2}}\right)_{x}+G\left(n, u_{2}\right), \\
& \frac{s}{\rho} u_{2 t}-\frac{\mu_{1}}{2} u_{2 x x t}-\mu u_{2 y y t}-\frac{1}{2} u_{2 x x}-(1+\nu) u_{2 y y}=\tau\left(\frac{n}{1+\alpha n^{2}}\right)_{y}+G\left(n, u_{1}\right), \\
& c_{t}=\gamma n-\delta \frac{n c}{K_{m}+c}+D_{2} \nabla^{2} c
\end{aligned}
$$

where

$$
G(n, v)=\left(\frac{1}{2}+\nu\right) v_{x y}-\left(\frac{\mu_{1}}{2}-\mu\right) v_{x y t}
$$

and $\mu=\mu_{1}+\mu_{2}$.

Equation (11) describing the cell density $n$ has reaction terms $(r n)$, advection terms (with advection velocity $\mathbf{v}=\left(u 1_{t}+\chi(c) c_{x}, u 2 t+\chi(c) c_{y}\right)$ ) and diffusion terms (where the $D_{i j}$ components of the diffusion tensor are given below).

Equation (12) is a conservation equation for the ECM density $\rho$.

Equations (13) and (14) originated from the description of the forces that act on the ECM. They describe the displacement $\mathbf{u}$ of the ECM and contain $\tau \frac{n}{1+\alpha n^{2}}$, a nonlinear term in $n$, which describes cell traction saturation at high densities.

Lastly, equation (15) is a reaction-diffusion equation for the chemical concentration, with nonlinear dynamics for the uptake of the chemical by the cells. 


\subsection{Boundary conditions}

As conditions at the boundary $\partial B$ we require that

- no cells cross the boundary of our domain (in the experiments, no cells go beyond the experimental dish sides):

- zero ECM flux across the boundary:

$$
\hat{\theta} \cdot J_{\text {cell }}=0
$$

$$
\hat{\theta} \cdot\left(\mathbf{u}_{t} \rho\right)=0
$$

- no chemical diffusion across the domain boundary:

$$
\hat{\theta} \cdot \nabla c=0
$$

- the ECM is attached to the dish sides, i.e. we have no ECM displacement at the boundary:

$$
\mathbf{u}=\mathbf{0},
$$

where $\hat{\theta}$ is the outward pointing normal at $\partial B$.

In [16] we also considered no cellular diffusion across the domain, which gives an alternative boundary condition for the cells:

$$
\hat{\theta} \cdot \nabla\left[\left(\begin{array}{cc}
D_{11} & D_{12} \\
D_{12} & D_{22}
\end{array}\right) n\right]=0,
$$

where

$$
\begin{aligned}
& D_{11}=\frac{D}{2}\left(2+u_{1 x}-u_{2 y}\right), \\
& D_{12}=\frac{D}{2}\left(u_{1 y}+u_{2 x}\right), \\
& D_{22}=\frac{D}{2}\left(2-u_{1 x}+u_{2 y}\right) .
\end{aligned}
$$

\section{NumericAl DiscRetization}

We will first describe the discretization methods employed for each of the five equations of the model, followed by the predictor-corrector scheme employed to solve these coupled equations.

We solve the equations numerically on square domains (their size is $\left[\begin{array}{ll}0 & 4\end{array}\right] \times\left[\begin{array}{ll}0 & 4\end{array}\right]$, unless otherwise stated). The domain is discretized into a uniform $m \times m$ Cartesian grid. By a point $(i, j)$ of the grid, we will mean the point in our domain with $x=i h, y=j h\left(h=\Delta x=\Delta y=\frac{4}{m}\right)$. By $n_{i j}$ we will denote the numerical approximation to the average value for the cell density $n$ on a square of dimensions $h \times h$ and center at the point $\left(i+\frac{1}{2}, j+\frac{1}{2}\right)$ of the grid. The values of $\rho_{i j}, u_{1 i j}, u_{2 i j}$ are defined the same way. The boundary lies along the lines $x=1+\frac{1}{2}, x=m+\frac{1}{2}$ and $y=1+\frac{1}{2}, y=m+\frac{1}{2}$.

\subsubsection{Discretization outline}

Equations (11) and (12) are discretized explicitely. The equation describing cells has terms that are parabolic or hyperbolic in character. We solve the equation by integrating each component of the equation separately, using a fractional step method.

Advection terms are discretized using upwinding, with second order, slope-limiter correction. The diffusion component, parabolic in character is usually solved using implicit methods. However, the parameter values that are relevant for the cell movement are small $\left(D \approx 10^{-11}\right)$, so that an explicit scheme for solving the parabolic equations suffices. 
Equations (13) and (14) for the displacement $\left(u_{1}, u_{2}\right)$ are discretized using an implicit scheme. Spatial derivatives are discretized with a centered in space scheme, time is discretized using the trapezoidal rule (TR), and the space and time discretization results in a Crank-Nicholson scheme.

\subsubsection{Time discretization}

The time discretization is governed by various conditions necessary for stability, as these arise in the discretizations of our equations. In general, the time point $r=N$ will correspond to real time $T_{N}=\sum_{r=1}^{r=N} k_{r}$ where $k_{r}$ will denote the time step considered at point $r$ in our time discretization. In particular, if $k_{N 1}, k_{N 2}$ are the time steps required for stability, as determined by each of the equations for $n, \rho$, then the time step $k_{N+1}$ from time point $r=N$ to the time point $r=N+1$ is determined by

$$
k_{N+1}=\min \left\{k_{N 1}, k_{N 2}, k_{m}\right\},
$$

where $k_{m}$ is the maximum allowable step in time.

\subsection{Matrix conservation equation}

We start with the discretization of equation (12) since it is the simplest one. This advection equation we discretize as

$$
\rho_{i, j}^{N+1}=\rho_{i, j}^{N}-\Delta t\left(\frac{f 1_{i+\frac{1}{2}, j}-f 1_{i-\frac{1}{2}, j}}{h}+\frac{f 2_{i, j+\frac{1}{2}}-f 2_{i, j-\frac{1}{2}}}{h}\right),
$$

where $f 1, f 2$ are the numerical approximations for the two components of the ECM flux $J_{E C M}=\left(u_{1 t} \rho, u_{2 t} \rho\right)$ and are defined as:

$$
f 1_{i+\frac{1}{2}, j}=v_{1 i+\frac{1}{2}, j}^{N} \rho_{i+\frac{1}{2}, j}^{N} .
$$

$f 2_{i, j+\frac{1}{2}}$ is defined similarly to $f 1_{i+\frac{1}{2}, j}$. In the above equation,

$$
v_{1 i+\frac{1}{2}, j}^{N}=\frac{1}{\Delta t}\left(u_{1 i+\frac{1}{2}, j}^{N+1}-u_{1 i+\frac{1}{2}, j}^{N}\right)
$$

is the velocity at $\left(i+\frac{1}{2}, j\right)$, defined implicitely in $u$, which means that we require knowledge of $u_{1 i+\frac{1}{2}, j}^{N+1}$ in order to determine $\rho_{i, j}^{N+1}$. We calculate $u_{1 i+\frac{1}{2}, j}^{N+1}$ via a predictor-corrector scheme which we present in Section 3.6.

Without loss of generality, when defining values at the half points, we can drop the index corresponding to the other direction, to keep notation simpler. The value at point $\left(i-\frac{1}{2}\right)$ is calculated using upwinding: we calculate the value based on the direction of the velocity $v$, which describes the direction information is coming from. We define

$$
\begin{array}{cl}
\rho_{i-\frac{1}{2}}=\rho_{i-1}+\frac{1}{2}(h-v k) \sigma_{i-1} & \text { if } \quad v>0 \\
\rho_{i}+\frac{1}{2}(h-v k) \sigma_{i} & \text { if } \quad v<0
\end{array}
$$

where

$$
\sigma_{i}=\sigma_{i}\left(\frac{\rho_{i+1}-\rho_{i}}{\Delta x}, \frac{\rho_{i}-\rho_{i-1}}{\Delta x}\right),
$$

is one of the standard limiters (MinMod, vanAlbada, van Leer).

To ensure stability for the advection step we impose the $C F L$ condition:

$$
\left|v^{N}\right| \frac{k_{N 1}}{\Delta x}<1
$$

where $v^{N}$ is the maximum velocity occuring at time $N$. 


\subsection{Cell conservation equation}

In discretizing (11) we use a time splitting:

- first solve the advection part of the equation, using first order upwinding, just as we did for the matrix conservation equation. The advective flux is a combination of the passive movement due to the matrix deformation $\left(\mathbf{u}_{t} n\right)$ and the cell movements along gradients of increasing chemical concentration $(\chi(c) \nabla(c) n)$.

We carry out the calculations over a full time step $\Delta t=k$ and arrive at an intermediate solution $n_{i, j}^{*}$ :

$$
n_{i, j}^{*}=n_{i, j}^{N}-\Delta t\left(\frac{g 1_{i+\frac{1}{2}, j}-g 1_{i-\frac{1}{2}, j}}{h}+\frac{g 2_{i, j+\frac{1}{2}}-g 2_{i, j-\frac{1}{2}}}{h}\right),
$$

where $(g 1, g 2)$ are the numerical approximations to the components of the cell flux

$$
\mathbf{J}_{c e l l}=\left(\left(\chi(c) c_{x}+u_{1 t}\right) n,\left(\chi(c) c_{y}+u_{2 t}\right) n\right) .
$$

$g 1_{i+\frac{1}{2}, j}$ represents the $x$ component of the cell flux at the midpoint between $(i+1, j)$ and $(i, j)$, and similarly for the other fluxes;

- we then apply the diffusion part by calculating it over time $\Delta t$. We discretize diffusion using a nine point stencil and we step in time applying forward Euler:

$$
n_{i j}^{N+1}=n_{i j}^{*}+D \frac{k}{h^{2}}\left(A_{x x}\left(D_{11, i, j} n_{i, j}^{*}\right)+2 A_{x y}\left(D_{12, i, j} n_{i, j}^{*}\right)+A_{y y}\left(D_{22, i, j} n_{i, j}^{*}\right)\right) .
$$

$A_{x x}, A_{x y}, A_{y y}$ are the standard central differencing operators, for example

$$
A_{x x} U_{i j}=\frac{1}{\Delta x^{2}}\left(U_{i-1, j}-2 U_{i j}+U_{i+1, j}\right)
$$

We chose the time step $\Delta t=k_{N 2}$ small enough so that $\frac{k_{N 2}}{h^{2}}\left|D_{i j}\right|<\frac{1}{2}$, for all $(i, j)$. Since the values of $D$ that are relevant are very small $\left(D_{i j} \leq 10^{-3}\right)$, the time step can be fairly large, depending on $h^{2}$. It turns out that the time step $k$ is determined by the CFL condition is considerably smaller than the time step required for stability in solving the diffusion part of the cell conservation equation. Numerical solution of the diffusion part of the cell conservation equation are shown in Figure 2;

- reaction terms are discretized using an explicit, forward Euler scheme.

When cell proliferation is taken into consideration, we can also apply a Strang splitting. If $\mathbf{A}_{\Delta t}, \mathbf{D}_{\Delta t}, \mathbf{R}_{\Delta t}$ are the solution operators for the advection, diffusion and reaction step respectively, to solve for $n^{N+1}$ we apply the operators as

$$
n^{N+1}=\mathbf{A}_{\Delta t / 2} \mathbf{D}_{\Delta t / 2} \mathbf{R}_{\Delta t} \mathbf{D}_{\Delta t / 2} \mathbf{A}_{\Delta t / 2} n^{N} .
$$

\subsection{Force balance equation}

The force balance equation yields

$$
\begin{gathered}
A u_{1 x x t}+B u_{1 y y t}+C u_{1 x x}+D u_{1 y y}+E u_{1 t}=F_{x}(n)+W u_{2 x y}+Y u_{2 x y t}, \\
A u_{2 y y t}+B u_{2 x x t}+C u_{2 y y}+D u_{2 x x}+E u_{2 t}=F_{y}(n)+W u_{1 x y}+Y u_{1 x y t},
\end{gathered}
$$

where

$$
\begin{array}{cccc}
A=\mu, & B=\frac{\mu_{1}}{2}, & C=\nu+1, & D=\frac{1}{2}, \\
E=-\frac{s}{\rho}, & F(n)=\frac{\tau n}{1+\alpha n^{2}}, & W=-\left(\frac{1}{2}+\nu\right), & Y=\left(\frac{\mu_{1}}{2}-\mu\right) .
\end{array}
$$

We discretize the above using Crank-Nicolson and get an $m-1 \times m-1$ system of equations of the form

$$
a_{i j} U 1_{i, j-1}^{N+1}+b_{i j} U 1_{i-1, j}^{N+1}+c_{i j} U 1_{i j}^{N+1}+d_{i j} U 1_{i+1, j}^{N+1}+e_{i j} U 1_{i, j+1}^{N+1}=q 1_{i j},
$$


with

$$
\begin{aligned}
a_{i j} & =\frac{1}{2 h^{2}}\left(\mu_{1}+\frac{k}{2}\right) \\
b_{i j} & =\frac{1}{h^{2}}\left(\mu+\frac{k}{2}(1+\nu)\right) \\
c_{i j} & =-\frac{2}{h^{2}}\left[\mu+\frac{k}{2}(1+\nu)+\frac{1}{2}\left(\mu_{1}+\frac{k}{2}\right)\right]-s\left(\bar{\rho}_{i j}^{N+1}\right)^{-1}, \\
d_{i j} & =\frac{1}{2 h^{2}}\left(\mu+\frac{k}{2}(1+\nu)\right) \\
e_{i j} & =\frac{1}{2 h^{2}}\left(\mu_{1}+\frac{k}{2}\right) .
\end{aligned}
$$

We employ an equivalent discretization for $u_{2}$.

Boundary conditions for the equations are $u_{i j}=0$ at boundary points and these values are incorporated in $q 1_{i j}$. The systems are solved using the NAG Mark 15, routine D03EBF, which solves equations of exactly the same form as (20). The routine derives the residual of the latest approximate solution and then uses the approximate $L U$ factorization of the Strongly Implicit Procedure, with the necessary acceleration parameter.

\subsection{Chemical reaction-diffusion equation}

In discretizing (15) we use once more a time splitting, whereby we solve diffusion and reaction terms separately. If $\mathbf{D} \mathbf{c}_{\Delta t}, \mathbf{R c}_{\Delta t}$ are the diffusion and reaction solution operators respectively for the chemical concentration, we calculate $c^{N+1}$ as

$$
c_{i j}^{N+1}=\mathbf{D} \mathbf{c}_{\Delta t / 2} \mathbf{R c}_{\Delta t} \mathbf{D} \mathbf{c}_{\Delta t / 2} c_{i j}^{N}
$$

\subsection{Initial conditions}

For the numerical simulations of cellular network formation we use the following initial conditions:

$$
\begin{aligned}
n_{i j}^{0} & =1.0 \pm 0.05 * \operatorname{rand}(i, j) \\
\rho_{i j}^{0} & =1.0 \\
\mathbf{u}_{i j}^{0} & =\mathbf{0} .
\end{aligned}
$$

The random numbers were generated by the built-in FORTRAN function ran, which distributes the random numbers uniformly in the interval $[0,1)$.

For the numerical simulation of sprout formation towards an angiogenic stimulus, we used as initial conditions:

$$
\begin{aligned}
n_{i j}^{0} & =1.0, \quad \text { if } \mathrm{i}<12 \text { or } \mathrm{j}>70, \\
& =0, \quad \text { otherwise, and } \\
\rho_{i j}^{0} & =1.0, \\
c_{i j}^{0} & =0, \\
\mathbf{u}_{i j}^{0} & =\mathbf{0} .
\end{aligned}
$$

\subsection{Predictor-corrector scheme - the algorithm}

On calculating $\rho_{i j}^{N+1}, n_{i j}^{N+1}$ we need to use values of $u_{1 i j}^{N+1}, u_{2 i j}^{N+1}$ while we have not yet solved for $u_{1}^{N+1}, u_{2}^{N+1}$. To overcome the problem, we use a prediction for the values of $u_{1}, u_{2}$, which we will denote by $u_{1 p}^{N+1}, u_{2 p}^{N+1}$. 

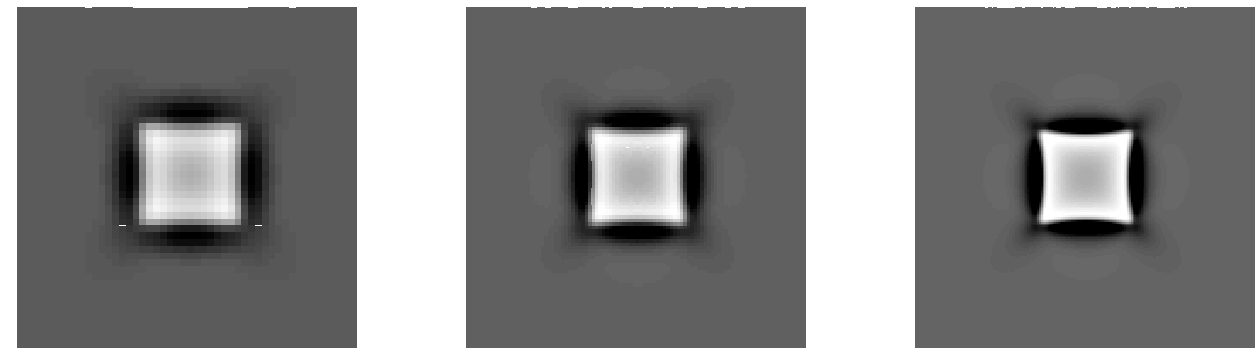

FIGURE 1. A square patch of cell density $n=1.1$ contracts, when the surrounding cell density is lower (here, it is $n=1$ ). From left to right, the grids used were: $50 \times 50,100 \times 100$ and $200 \times 200$, all uniform Cartesian grids. Parameter values for the model equations are given in Table 2 .

The values for $\rho_{i j}^{N+1}, n_{i j}^{N+1}$ thus calculated are not accurate, since their calculation involved predicted, not calculated values of the other dependent variables. This inaccuracy will affect the further calculations. To solve the problem, we use a predictor-corrector scheme, whereby we iterate a few times within the same time step through the equation solvers, until successive calculations for one of the dependent variables, say $u_{1}$, are equal within a certain error tolerance.

This predictor corrector scheme between time step $N$ and $N+1$ is expressed by the following steps:

(1) predict the values for $u_{1 p}^{N+1}, u_{2 p}^{N+1}$, using some extrapolation method;

(2) use $u_{1 p}^{N+1}, u_{2 p}^{N+1}$ to get approximate values for $n^{* N+1}, \rho^{* N+1}$;

(3) use $n^{* N+1}, \rho^{* N+1}$ to get a better approximation for $u_{1}^{N+1}, u_{2}^{N+1}$;

(4) continue the iterations until the difference between successive approximations of $n$, say, are less than a tolerance: $\Delta n=\left|n^{* N+1}-n^{* * N+1}\right|<\delta$.

\subsection{Convergence and accuracy of the methods employed}

\subsubsection{Convergence}

The model equations are nonlinear and therefore standard techniques for verifying the stability (Von Neumann analysis) and accuracy of the model are not applicable. In this paper we shall examine only the computational stability required for our algorithm to work properly. Instead, we perform various tests on the discretized model to check its robustness. We consider the program predictions when the mesh size of a fixed size domain is varied. In Figure 1, we compare a square of cells evolving in a $50 \times 50$ grid with mesh size $h=0.08$, a $100 \times 100$ grid with mesh size $h=0.04$ and on a $200 \times 200$ grid with mesh size $h=0.02$. The size of the central square is $1.12 \times 1.12$. We observe a fairly good agreement in the solutions that appear with the three discretizations.

\subsubsection{Accuracy}

We employ a combination of schemes, some first order in time (forward Euler) and others second order in time (trapezoidal). We therefore expect our scheme to be approximately first order accurate in time. For the spatial derivatives we have employed second order discretizations.

\subsubsection{Conservation of mass}

In the absence of cell multiplication, equation (11) is a conservation equation for the cell density. To test the code, we kept track of the total cell density within our domain and found that to be conserved to machine epsilon.

\subsubsection{Cell diffusion}

Through simple numerical simulations, we test the behavior of the discretization of the diffusion operator. 


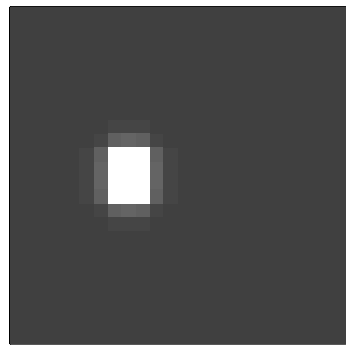

A

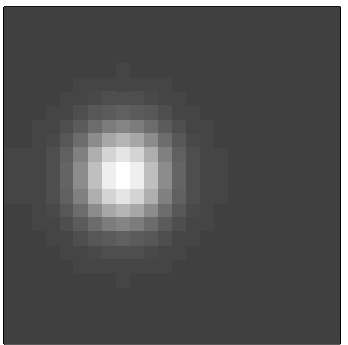

B

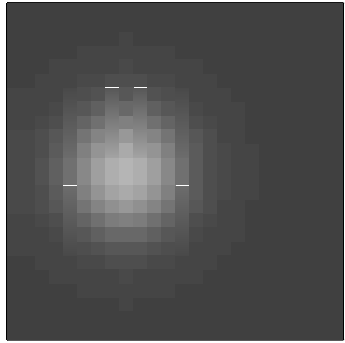

C

Figure 2. A square patch of cells diffusing on an unstrained matrix. Parameter values are given in Table 2.
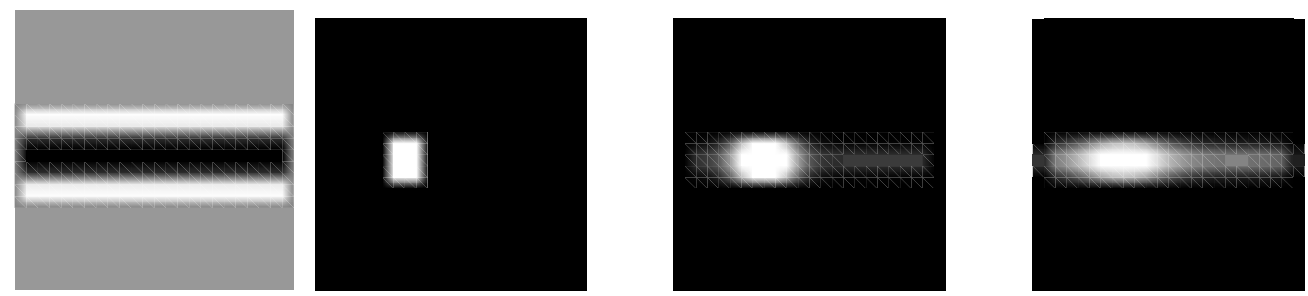

Figure 3. Cells diffusing along areas of matrix compression. The strain field is shown in the left picture. Gray denotes unstrained areas, Black denotes compression and white denotes stretched (expanded) matrix. Parameter values are given in Table 2.

TABLE 2. Dimensional model parameters simulation parameters for the test simulation figures, unless stated otherwise.

\begin{tabular}{|c|c|c|c|}
\hline Model parameters & Value & Simulation parameters & \\
\hline Traction $\tau$ & 0.005 dyne/cell & Max. time step $k_{\max }$ & 0.2 \\
\hline$\alpha$ & $10^{-9} /$ cell $^{2}$ & Initial time step $k_{0}$ & $10^{-4}$ \\
\hline Diffusion $D$ & $0.7 \times 10^{-12} \mathrm{~cm}^{2} / \mathrm{sec}$ & Grid & $50 \times 50$ \\
\hline Poisson ratio $\nu$ & 0.2 & Mesh size & 0.1 \\
\hline Shear viscosity $\mu_{1}$ & $3.2 \times 10^{7}$ poise & \multirow{2}{*}{$\begin{array}{l}\text { Error tolerance between } \\
\text { successive solution estimates }\end{array}$} & \multirow[b]{2}{*}{$10^{-14}$} \\
\hline Bulk viscosity $\mu_{2}$ & $6.5 \times 10^{7}$ poise & & \\
\hline Youngs Modulus $E$ & 20 dyne $/ \mathrm{cm}^{2}$ & & \\
\hline Anchoring parameter $s$ & $10^{10}$ dynes $\cdot \mathrm{sec} / \mathrm{cm}^{3}$ & & \\
\hline
\end{tabular}

We consider cells on a fixed, unstrained matrix. We hold the matrix fixed at its original position for all times and let the cells perform an unbiased diffusive motion. The results are presented in Figure 2.

We also consider cells in a strained environment. We should expect cells to move along compressed areas. Figure 3 shows an initial square patch of cells diffusing along a line of compression. The strain field is shown on the leftmost picture. Black denotes areas of compression and white denotes areas of dilation. Gray areas are unstrained. We observe that the cells move along the line of compression. 


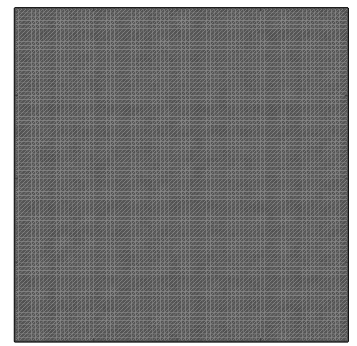

$t=4$

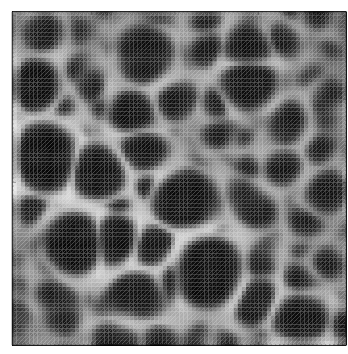

$t=16$

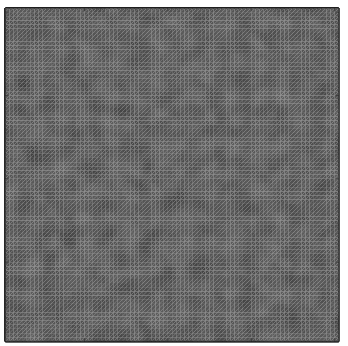

$t=8$

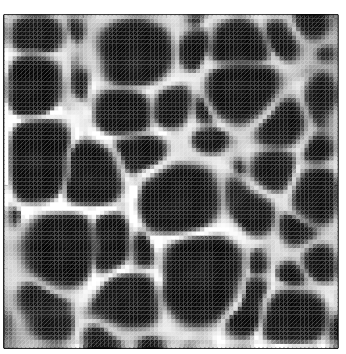

$t=20$

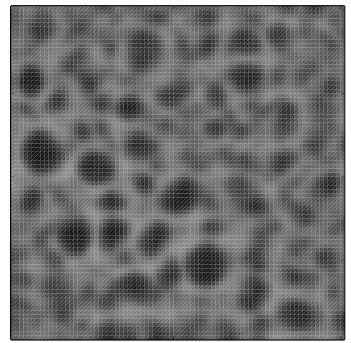

$\mathrm{t}=12$

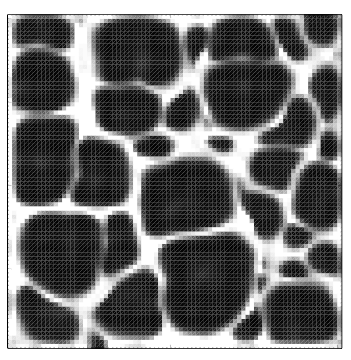

$t=24$

FiguRE 4. Numerical simulation of vascular network formation. Cells reorganize into networks, assuming a purely mechanical interaction between cells and the ECM (i.e. chemotaxis is not taken into account in the above simulation). Initially cells are approximately uniform, but slowly areas of higher cell densities form (white) which exert traction onto the matrix and generate areas devoid of cells (black). Simulations were performed on an $80 \times 80$ uniform grid, corresponding to a square $3.2 \times 3.2 \mathrm{~mm}^{2}$. Here, we assume no diffusive cell flux across the boundary. The patterns do not reach a steady state, but continue growing, with some capillary loops growing larger and others closing in, in a sphincter-like manner. Parameter values: $\tau=$ 0.01 dynes $/$ cell, $E=20$ dyne $/ \mathrm{cm}^{2}, \nu=0.2, \mu_{1}=1.4 \times 10^{7}$ poise, $\mu_{2}=1.2 \times 10^{8}$ poise, $n_{0}=$ $10^{4}$ cells $/ \mathrm{cm}^{2}$.

\section{Results}

Mechanical forces lead to formation of networks

During vascular network formation, cells that are initially distributed throughout the domain of concern, reorganize themselves into networks. Vernon et al. proposed that the cells move and the ECM reorganizes into a network under the influence of cellular forces $[25,26]$.

To test the hypothesis, we consider only the mechanical interactions within the model and ignore cell movement under chemical gradients as well as cell proliferation. We model the initial condition as an almost uniform distribution of cells, matrix, chemical density, throughout the domain. Numerical results, shown in Figure 4, suggest formation of cellular networks that compare very well with experimentally observed networks (see, for example $[25,26])$.

We assume that the pattern forming mechanism works as follows: A small (random) perturbation in the otherwise uniform cell population creates local gradients in the cell density. As the total traction in one point is 


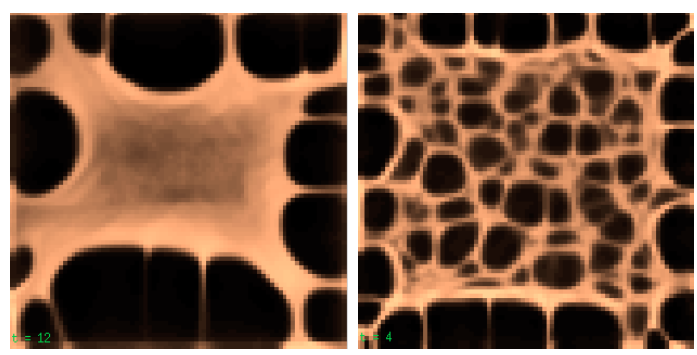

FIGURE 5. Left: If traction is too low, cells will contract towards the center of the domain. Right: If traction is sufficiently high (as predicted by linear stability analysis), networks will form. The simulation results assume zero cell flux as the boundary condition. Simulations were performed on an $80 \times 80$ grid, total side length $3.2 \mathrm{~mm}$. Parameter values: $\tau=0.01$ dynes/cell (right), $\tau=0.005$ dynes $/$ cell (left), $E=20$ dyne $/ \mathrm{cm}^{2}, \nu=0.2, \mu_{1}=5.8 \times 10^{8}$ poise, $\mu_{2}=2$ $\times 10^{8}$ poise, $n_{0}=10^{4}$ cells $/ \mathrm{cm}^{2}$.

proportional to the cell density, the gradient in $n$ will result in a net force, with direction from lower to higher cell density. This force is resisted by the mechanical behavior of the ECM. As we have shown before [16, 19], if the cell traction is large enough to overcome the resistance by the ECM stiffness, cells will move from regions of lower density to regions of higher density, thus generating small perforations in the collagen and cell layer. The movement will start at a small scale, and, as the clusters grow, so will the areas devoid of cells, until we get a network of cells. If the ECM is too stiff, then no pattern will form.

The above claim is confirmed by linear stability analysis: We assume solutions to the model equations of the form

$$
(n, \rho, \mathbf{u}, c)=\left(n_{0}, \rho_{0}, \mathbf{u}_{0}, c_{0}\right)+\left(n^{*}, \rho^{*}, \mathbf{u}^{*}, c^{*}\right) e^{\sigma t+i \mathbf{k} \mathbf{x}},
$$

where the asteriscs denote small quantities, i.e. we assume the variables near the uniform steady state $\left(n_{0}, \rho_{0}\right.$, $\mathbf{u}_{0}, c_{0}$ ) will grow or decay exponentially.

If we substitute the above into the equations, and ignoring small terms $\left(n^{* 2}, u_{1}^{* 2}\right.$, etc.), we get the dispersion relation, which associates different wavenumbers $\mathbf{k}$ with their corresponding growth rate $\sigma\left(\mathbf{k}^{2}\right)$. For pattern to grow we require that at least for certain $\mathbf{k}$ the corresponding growth rate is positive, i.e. the initial small perturbation will grow, giving rise to pattern. Using the method presented in [16] it can be shown that we will have positive growth rates if

$$
\frac{\tau}{E}>\frac{1}{1-\nu^{2}} \frac{1}{n_{0}} \frac{\left(1+a n_{0}^{2}\right)^{2}}{1-\alpha n_{0}^{2}},
$$

where $E$ is the matrix stiffness, $\tau$ is the traction per cell, $\nu$ is the Poisson ratio, $n_{0}$ is the initial uniform cell density, and $\alpha$ determines the decrease in total cell traction upon confluence. The result suggests that pattern can form if cellular traction $\tau$ is sufficiently high or matrix stiffness $E$ sufficiently low. If cellular traction is too low no networks form (Fig. 5). The kinetic parameters of chemical production or uptake do not contribute to a positive growth rate of an initial perturbation. We do not mean that chemotaxis is not important. It plays a key role in the sprouting of endothelial cells from extant vasculature and its role has been discussed at length in mathematical models of angiogenesis (e.g. $[2,5])$. What our results suggest is that chemotaxis alone is not a sufficient force to stimulate formation of pattern if we start with a perturbation about the uniform initial steady state, a situation that is a closer model for vasculogenesis, rather than angiogenesis (sprouting).

Cell chemotaxis leads to sprout formation

Other models of angiogenesis also suggest that sprout development towards a source of chemoattractant cannot be resolved by assuming only chemotaxis of cells and that other mechanisms also come into effect $(e . g$. haptotaxis, [5], cellular inhibition [18], etc.). Here we test the combination of chemotaxis with mechanical forces as a minimum mechanism for angiogenic sprout formation. 


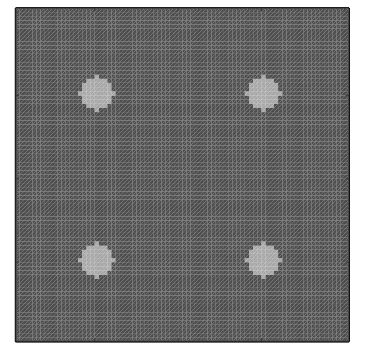

$t=1$

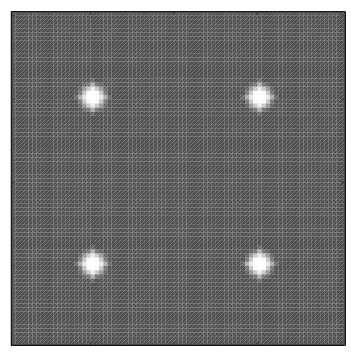

$\mathrm{t}=1$

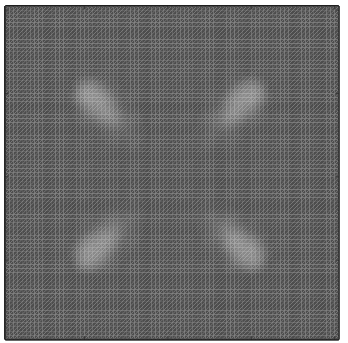

$t=15$

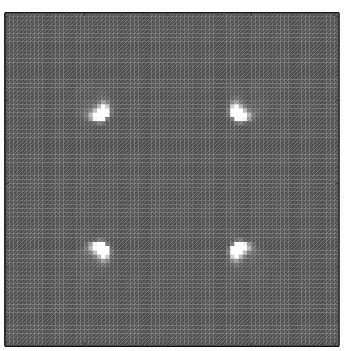

$\mathrm{t}=15$

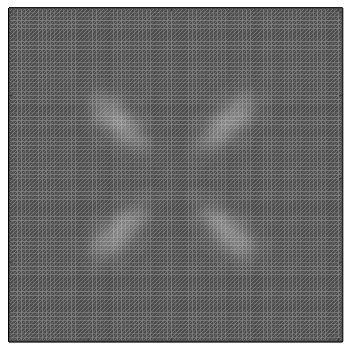

$t=30$

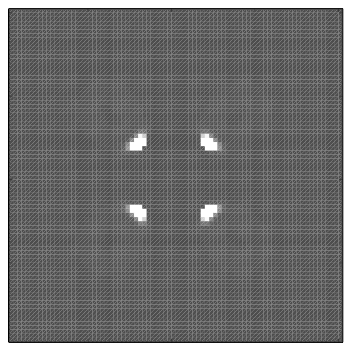

$\mathrm{t}=30$

Figure 6. If cells are made unable to pull (top figure), four cell clusters placed symmetrically around a central source of chemoattractant (positioned at the center of the domain), will move up gradients of increasing chemoattractant density. If cells can also exert traction (bottom figure), the cells will form more concentrated clusters and will move faster towards the chemoattractant source. In the absence of chemoattractant, cell clusters will move very little or not at all, depending on the distance between neighbouring clusters (not shown).

In the presence of a chemoattractant source, cells move up the gradient of a chemoattractant (Fig. 6), and cell traction accelerates the movement of the cell clusters towards each other.

Previous models of chemically-driven angiogenesis simulate growth of vessels that starts out from pre-existing sprouts (see, for example [2,12]). Here, we consider an inital uniform band of cells, which models a vessel placed near a source of chemoattractant. We observe that at the point nearest to the chemoattractant source, the cells start moving towards the source. When no cell traction is included, cells from a wide section of the original vessel migrate towards the chemical source. When cell traction was included, the band of cells initially contracted due to cell-exerted traction. After some time (nondimensional time $t=18$ ), cells sprout from the parent vessel and slowly grow towards the center, forming better defined, narrower structures (Fig. 7).

\section{Conclusion}

We presented a mathematical model for endothelial cells-ECM interactions during the initial stages of blood vessel formation. The model describes the traction the cells exert onto the ECM and models the ECM response as that of a linear viscoelastic material. We assumed cells can move passively, when they adhere on moving ECM, do strain-biased random walk, and move along chemical gradients. 


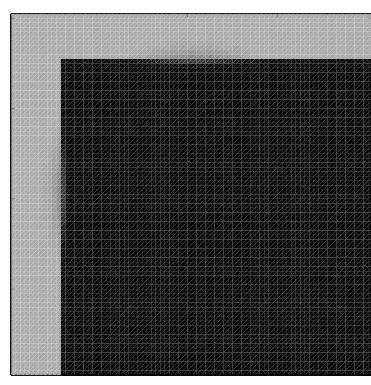

$\mathrm{t}=6$

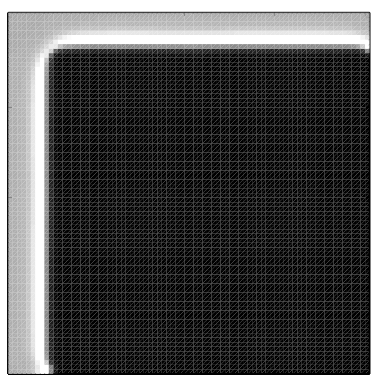

$t=6$

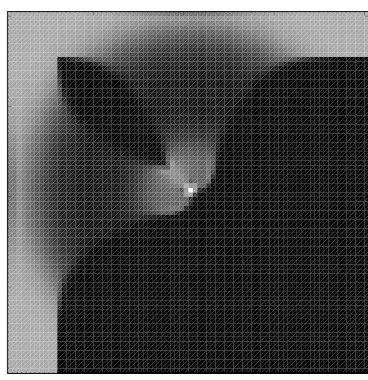

$t=21$

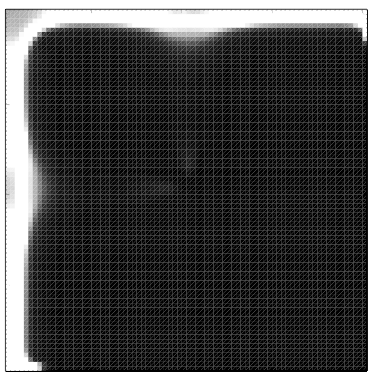

$t=21$

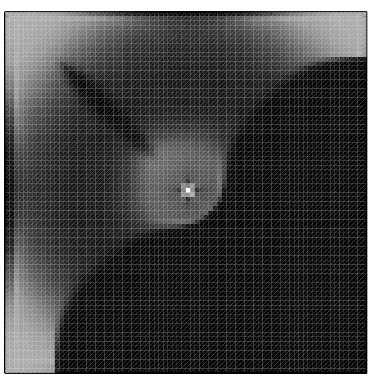

$t=36$

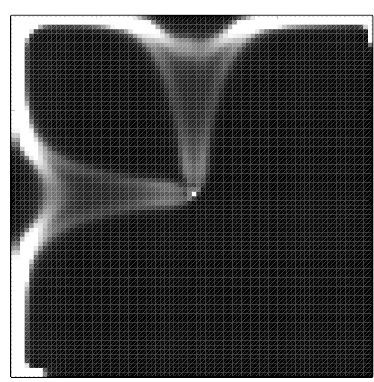

$t=36$

FiguRE 7. Starting from two continuous bands of cells, placed on the left and top of the figure, cells will move towards a centrally placed source of chemoattractant. In the top figure, no cell traction is assumed. In the lower figure, the bands of cells initially contract a little, under cell-exerted traction. Cells sprout slowly $(t=18)$ and grow towards chemoattractant source. The structures formed are narrower, and better defined when cellular traction is considered (cell traction values same as in Figure 4, chemotactic parameters provided in Table 3).

TABLE 3. Nondimensional parameter values for the simulation results with chemotaxis.

\begin{tabular}{|l|c|}
\hline Model parameters & Value \\
\hline \hline$\chi_{0}$ & 0.25 \\
\hline$D_{2}$ & 0.02 \\
\hline$\beta$ & 0 \\
\hline$\gamma$ & 0 \\
\hline$\delta$ & 0.0065 \\
\hline
\end{tabular}

We studied the equations numerically: we presented an algorithm that was based on a fractional step method, whereby we treated advection, diffusion and reaction terms of the equations separately, using suitable methods. 
When we simulated cellular movement in the absense of potential cell interactions with chemical gradients and no cell proliferation, we predicted formation of network structures (Fig. 4) that resemble the cellular networks observed in in vitro experiments as well as examples of early planar vasculature. The agreement of the networks predicted by our model with those of in vitro models of vasculogenesis suggest that cellular networks could form via a purely mechanical mechanism in these early stages of vascular development.

Further remodelling of the plexus is determined by the chemotactic response of endothelial cells to angiogenic stimuli. Our model confirmed the work of other researchers in that it showed chemotaxis alone is not sufficient in giving rise to new vasculature and that a mechanical interaction with the ECM is necessary. The difference in our model is that the only mechanical interaction we consider is the traction of the cells onto the ECM and the ECM viscoelastic response. Our model predicts that chemical gradients together with cellular traction can make cells sprout away from the parent vessel and towards a source of chemoattractant. We did not predict capillary anastomoses, confirming what others have shown, that regulation of the cell movement and adhesion dynamics via fibronectin is likely to play an important role in the mechanism for capillary loop formation [2,5].

In summary, we presented a model that showed that a simple mechanical and chemical interaction was able to predict the basic features of the two main mechanisms (angiogenesis and vasculogenesis) of blood vessel formation.

Acknowledgements. The author would like to thank professors Peter J. Schmid (University of Washington) and Theodoros Katsaounis (University of Crete) for their valuable suggestions and remarks with the numerical methods.

\section{REFERENCES}

[1] S.G. Advani and C.L. Tucker, The use of tensors to describe and predict fiber orientation in short fiber composites. J. Rheol. 31 (1987) $751-784$

[2] A.R. Anderson and M.A. Chaplain, Continuous and discrete mathematical models of tumor-induced angiogenesis. B. Math. Biol. 60 (1998) 857-900.

[3] D.H. Ausprunk and J. Folkman, Migration and proliferation of endothelial cells in preformed and newly formed blood vessels during tumour angiogenesis. Microvasc. Res. 14 (1977) 53-65.

[4] M.A. Chaplain, Mathematical modelling of angiogenesis. J. Neuro. 50 (2000) 37-51.

[5] M.A. Chaplain and A.R. Anderson, Mathematical modelling, simulation and prediction of tumour-induced angiogenesis. Invasion Metastasis 16 (1996) 222-234.

[6] J. Cook, Mathematical Models for Dermal Wound Healing: Wound Contraction and Scar Formation. Ph.D. thesis, University of Washington (1995).

[7] C.J. Drake and A.G. Jacobson, A survey by scanning electron microscopy of the extracellular matrix and endothelial components of the primordial chick heart. Anat. Rec. 222 (1988) 391-400.

[8] C.J. Drake and C.D. Little, The morphogenesis of primordial vascular networks. In Vascular Morphogenesis: In Vivo, In Vitro, In Mente, Charles D. Little, Vladimir Mironov and E. Helene Sage Eds., Chap. 1.1, Birkauser, Boston, MA (1998) 3-19.

[9] J. Folkman and C. Haudenschild, Angiogenesis in vitro. Nature 288 (1980) 551-556.

[10] E.A. Gaffney, K. Pugh, P.K. Maini and F. Arnold, Investigating a simple model of cutaneous would healing angiogenesis. J. Math. Biol. 45 (200) 2337-374.

[11] D. Hanahan, Signaling vascular morphogenesis and maintenance. Science 227 (1997) 48-50.

[12] M.J. Holmes and B.D. Sleeman, A mathematical model of tumor angiogenesis incorporating cellular traction and viscoelastic effects. J. Theor. Biol. 202 (2000) 95-112.

[13] Y. Lanir, Constitutive equations for fibrous connective tissues. J. Biomech. 16 (1983) 1-12.

[14] H.A. Levine, B.D. Sleeman and M. Nilsen-Hamilton, Mathematical modeling of the onset of capillary formation initiating angiogenesis. J. Math. Biol. 42 (2001) 195-238.

[15] D. Manoussaki, Modelling the formation of vascular networks in vitro. Ph.D. thesis, University of Washington (1996).

[16] D. Manoussaki, S.R. Lubkin, R.B. Vernon and J.D. Murray, A mechanical model for the formation of vascular networks in vitro. Acta Biotheoretica 44 (1996) 271-282.

[17] R.R. Markwald, T.P. Fitzharris, D.L. Bolender and D.H. Bernanke, Sturctural analysis of cell: matrix association during the morphogenesis of atrioventricular cushion tissue. Developmental Biology 69 (1979) 634-54.

[18] H. Meinhardt, Models for the formation of netline structures, in Vascular Morphogenesis: In Vivo, In Vitro, In Mente, Charles D. Little, Vladimir Mironov and E. Helene Sage Eds., Chap. 3.1, Birkauser, Boston, MA (1998) $147-172$. 
[19] J.D. Murray, D. Manoussaki, S.R. Lubkin and R.B. Vernon, A mechanical theory of in vitro vascular network formation, in Vascular Morphogenesis: In Vivo, In Vitro, In Mente, Charles D. Little, Vladimir Mironov and E. Helene Sage Eds., Chapter 3.2, Birkauser, Boston, MA (1998) 173-188.

[20] J.D. Murray, G.F. Oster and A.K. Harris, A mechanical model for mesenchymal morphogenesis. J. Math. Biol. 17 (1983) $125-129$.

[21] G.F. Oster, J.D. Murray and A.K. Harris, Mechanical aspects of mesenchymal morphogenesis. J. Embryol. Exp. Morph. 78 (1983) 83-125.

[22] L. Pardanaud, F. Yassine and F. Dieterlen-Lievre, Relationship between vasculogenesis, angiogenesis and haemopoiesis during avian ontogeny. Development 105 (1989) 473-485.

[23] W. Risau, H. Sariola, H.G. Zerwes, J. Sasse, P. Ekblom, R. Kemler and T. Doetschmann, Vasculogenesis and angiogenesis in embryonic-system-cell-derived embryoid bodies. Development 102 (1988) 471-478.

[24] S. Tong and F. Yuan, Numerical simulations of angiogenesis in the cornea. Microvasc. Res. 61 (2001) 14-27.

[25] R.B. Vernon, J.C. Angello, M.L. Iruela-Arispe, T.F. Lane and E.H. Sage, Reorganization of basement membrane matrices by cellular traction promotes the formation of cellular networks in vitro. Lab. Invest. 66 (1992) 536-547.

[26] R.B. Vernon, S.L. Lara, C.J. Drake, M.L. Iruela-Arispe, J.C. Angello, C.D. Little, T.N. Wight and E.H. Sage, Organized type I collagen influences endothelial patterns during "spontaneous angiogenesis in vitro": Planar cultures as models of vascular development. In Vitro Cellular and Developmental Biology 31 (1995) 120-131.

[27] R.B. Vernon and E.H. Sage, Between molecules and morphology: extracellular matrix and the creation of vascular form. $A m$. J. Pathol. 147 (1995) 873-883. 